\title{
Racemic charge-transfer complexes of a helical polycyclic aromatic hydrocarbon molecule
}

\section{$\operatorname{AUTHOR}(\mathrm{S})$ :}

Yoshida, Yukihiro; Nakamura, Yuto; Kishida, Hideo; Hayama, Hiromi; Nakano, Yoshiaki; Yamochi, Hideki; Saito, Gunzi

\section{CITATION:}

Yoshida, Yukihiro ...[et al]. Racemic charge-transfer complexes of a helical polycyclic aromatic hydrocarbon molecule. CrystEngComm 2017, 19(26): 3626-3632

\section{ISSUE DATE:}

2017-06-02

\section{URL:}

http://hdl.handle.net/2433/230179

\section{RIGHT:}

This is the accepted version of the article, which has been published in final form at http://doi.org/10.1039/c7ce00763a; The full-text file will be made open to the public on 01 Jun 2018 in accordance with publisher's 'Terms and Conditions for Self-Archiving'; This is not the published version. Please cite only the published version.; この論文は出版社版であり ません。引用の際には出版社版をご確認ご利用ください。 


\section{CrystEngComm}

\section{Racemic Charge-Transfer Complexes of Helical Polycyclic Aromatic Hydrocarbon Moleculet}

Received 00th January 20xx, Accepted 00th January 20xx

DOI: $10.1039 / x 0 x \times 00000 x$

www.rsc.org/

\author{
Yukihiro Yoshida, ${ }^{\text {ab }}$ Yuto Nakamura, ${ }^{\mathrm{c}}$ Hideo Kishida, ${ }^{\mathrm{c}}$ Hiromi Hayama, ${ }^{\mathrm{a}}$ Yoshiaki Nakano, ${ }^{\text {bd }}$ Hideki \\ Yamochi $^{\text {bd }}$ and Gunzi Saito ${ }^{\text {ae }}$
}

Singles crystals of racemic charge-transfer complexes comprising a helical polycyclic aromatic hydrocarbon molecule, [6]helicene, as the electron donor (D) and 7,7,8,8-tetracyano- $p$-quinodimethane (TCNQ) analogs as the electron acceptor (A) were obtained by either co-sublimation or solvent evaporation. All complexes consist of heterochiral DA-type alternating $\pi$-columns with a $[\cdots(P)$-[6]helicene $\cdots$ TCNQ $\cdots(M)$-[6]helicene $\cdots$ TCNQ $\cdots]$ repeating unit, which show a peculiar wavy feature primarily related to the nonplanar geometry of [6] helicene molecules. [6] Helicene molecules form a two-dimensional sheet intersecting the columns, and the molecular arrangement (namely, either homochiral or heterochiral arrangement) in the sheets depends on the TCNQ analog. Theoretical calculations were carried out to discuss the origin of the molecular deformation of [6]helicene molecules in the CT solids in terms of intermolecular interactions.

\section{Introduction}

For more than half a century, polycyclic aromatic hydrocarbons (PAHs) have been widely utilised in organic electronics, e.g., photovoltaics, field-effect transistors, and solar cells. ${ }^{1-3}$ Intermolecular $\pi-\pi$ interactions promote the self-assembly of PAH molecules into columnar stacks; therefore, these interactions play a crucial role in the $\pi$-electronic properties of the aggregates. Furthermore, co-crystallization with electron-donating (D) or accepting (A) counter molecules, with diverse combinations, utilizing charge-transfer (CT) interactions has afforded a variety of exotic PAH-based electronic materials, e.g., metals and superconductors. ${ }^{4-6}$

Recently, nonplanar $\pi$-conjugated molecules have attracted attention as components for the assembly of an unconventional $\pi$ electron network. ${ }^{7-9}$ Two main approaches are utilised to produce nonplanar PAH molecules: endoskeletal and exoskeletal approaches. In the former approach, a nonhexagonal ring is present inside hexagonal skeletons (e.g., $[n]$ circulenes; ${ }^{10}$ Scheme 1a), while the latter approach exploits steric hindrance because of crowdedness (e.g., $[n]$ helicenes; ${ }^{11,12}$ Scheme $\left.1 \mathrm{~b}\right)$. With respect to the former type, our group has reported the first corannulene-based CT solid using 7,7,8,8tetracyano- $p$-quinodimethane $\quad$ (TCNQ; Scheme 1c), (corannulene) 2 TCNQ; in this solid, the CT interaction with an adjacent TCNQ molecule on the convex side of the corannulene is apparently greater than that on the concave side. ${ }^{13}$ In this study, four

\footnotetext{
a. Faculty of Agriculture, Meijo University, Nagoya 468-8502, Japan; E-mail yyoshida@meijo-u.ac.jp

b. Division of Chemistry, Graduate School of Science, Kyoto University, Kyoto 6068502, Japan

c. Department of Applied Physics, Nagoya University, Nagoya 464-8603, Japan

d. Research Center for Low Temperature and Materials Sciences, Kyoto University, Sakyo-ku, Kyoto 606-8501, Japan

e. Toyota Physical and Chemical Research Institute, Nagakute 480-1192, Japan

+ Electronic Supplementary Information (ESI) available: Infrared spectra and X-ray crystallographic data in CIF format for 1-4. See DOI: 10.1039/x0xx00000x
}

racemic $\mathrm{CT}$ complexes based on [6] helicene, which is categorised into the latter group, were prepared using various TCNQ analogs (Scheme 1c-1f), and their optical and crystallographic properties, in addition to theoretical calculations, were investigated. For $[n]$ helicenes, increasing efforts continue to be devoted to achieving control crystalline forms of racemates, i.e., racemic mixtures (conglomerates) racemic compounds, or solid solutions, by changing molecular structures, counter components, and crystallization processes. ${ }^{11,12}$ (a)

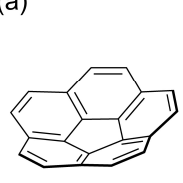

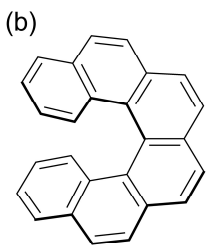

(c)<smiles>N#CC(C#N)=c1ccc(=C(C#N)C#N)cc1</smiles>

(d)<smiles>N#CC(C#N)=c1c(F)c(F)c(=C(C#N)C#N)c(F)c1F</smiles>

(e)<smiles>N#CC(C#N)=c1cc(F)c(=C(C#N)C#N)cc1F</smiles><smiles>Cc1cc(=C(C#N)C#N)c(=C(C#N)C#N)cc1C</smiles>

Scheme 1 Molecular structures of (a) corannulene ([5]circulene),

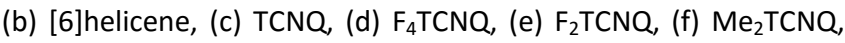
and (g) $\mathrm{Me}_{4} \mathrm{TCNQ}$.

\section{Results and Discussion}

\section{Crystal Growth}

Commercial racemic [6] helicene was used as received. CT complexes with TCNQ analogs, ([6]helicene)(F4TCNQ) (1) ([6]helicene)(F2TCNQ) (2), and ([6]helicene)(Me2TCNQ) (4), were prepared by co-sublimation, whereas a CT complex with parent 

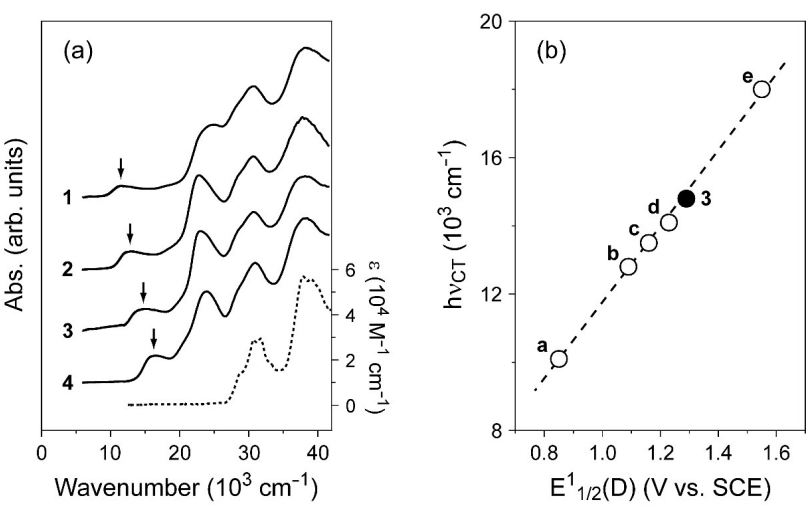

Fig. 1 (a) Electronic absorption spectra of 1-4 measured on $\mathrm{KBr}$ pellets (solid lines) together with pristine [6] helicene in a $10^{-5} \mathrm{M}$ chloroform solution (dotted line). Arrows denote the CT absorption bands. (b) Plot of CT energy $\left(h v_{C T}\right)$ against the first redox potential of PAH molecules $\left(E_{1 / 2}^{1}(D)\right)$ in the TCNQ complexes (a: (perylene)(TCNQ), ${ }^{17} \mathbf{b}$ : (anthracene)(TCNQ), ${ }^{18}$ c: (pyrene)(TCNQ), ${ }^{19}$ d: (coronene)(TCNQ), ${ }^{20,21}$ e: (triphenylene) ${ }_{2}(\mathrm{TCNQ})$ (toluene) ${ }^{22}$ ).
TCNQ, ([6]helicene)(TCNQ) (3), ${ }^{14}$ was prepared by solvent evaporation (see Experimental section). Notably, co-sublimation and solvent evaporation using $\mathrm{Me}_{4} \mathrm{TCNQ}$ (Scheme $1 \mathrm{~g}$ ) with a weak electron-accepting ability $\left(E^{1}{ }_{1 / 2}(\mathrm{~A})=-0.05 \mathrm{~V}\right.$ vs. saturated calomel electrode (SCE)) afforded no trace of the CT complex.

\section{Charge Transfer}

Fig. 1a shows the electronic absorption spectra of 1-4 dispersed in $\mathrm{KBr}$ pellets. A low-lying band, corresponding to the intermolecular CT transition, was observed at $h v_{\mathrm{CT}}=11-17 \times 10^{3} \mathrm{~cm}^{-1}$ in addition to the intramolecular transition bands of the component molecules at greater than $20 \times 10^{3} \mathrm{~cm}^{-1}$. The low-energy band provides clear evidence of the CT interactions between [6] helicene and TCNQ analogs in solids. The $h v_{\mathrm{CT}}$ value steadily decreases with the increase in the electron-accepting ability of the TCNQ analogs (Table 1). This relationship is indicative of a neutral ground state with alternating $\pi$ columns, ${ }^{15}$ which is in agreement with the high first redox potential of [6] helicene $\left(E^{1}{ }_{1 / 2}(\mathrm{D})=1.29 \mathrm{~V}\right.$ vs. $\mathrm{SCE}^{16}$ relative to the $E^{1}{ }_{1 / 2}(\mathrm{~A})$ values of the TCNQ analogs. Notably, complex 3 formed with parent TCNQ lies on a straight line via the application of a least-squares method to the plots of $h v_{\mathrm{CT}}$ against $E^{1}{ }_{1 / 2}$ (D) of typical planar PAH

Table 1 Typical parameters for $\mathbf{1 - 4}$

\begin{tabular}{|c|c|c|c|c|c|}
\hline Complex & 1 & 2 & 3 & 4 & (M)-[6]helicene \\
\hline Acceptor (A) & $\mathrm{F}_{4} \mathrm{TCNQ}$ & $\mathrm{F}_{2} \mathrm{TCNQ}$ & TCNQ & $\mathrm{Me}_{2} \mathrm{TCNQ}$ & - \\
\hline$E^{1}{ }_{1 / 2}(\mathrm{~A})(\mathrm{V} \text { vs. SCE })^{a}$ & 0.60 & 0.41 & 0.22 & 0.15 & - \\
\hline$h v_{\mathrm{CT}}\left(10^{3} \mathrm{~cm}^{-1}\right)^{b}$ & 11.6 & 12.9 & 14.8 & 16.5 & - \\
\hline$d(\AA \AA)^{c}$ & 4.39 & 4.20 & $4.19,4.20$ & 4.12 & 4.48 \\
\hline$\gamma\left({ }^{o}\right)^{d}$ & 57.6 & 47.0 & $45.1,46.2$ & 41.8 & 59.5 \\
\hline
\end{tabular}

${ }^{a}$ First redox potential of TCNQ analogs (SCE: saturated calomel electrode). ${ }^{b} \mathrm{CT}$ absorption band measured on $\mathrm{KBr}$ pellets. ${ }^{c} \mathrm{Distance}$ between the terminal benzene centroids in a [6] helicene molecule based on the crystallographic data at $100 \mathrm{~K} .{ }^{d}$ Angle between the planes of the terminal benzene rings in a [6]helicene molecule based on the crystallographic data at $100 \mathrm{~K}$.

Table 2 Crystallographic data of 1-4

\begin{tabular}{|c|c|c|c|c|c|}
\hline Complex & 1 & 2 & 3 & 4 & $(M)$-[6]helicene \\
\hline Formula & $\mathrm{C}_{38} \mathrm{H}_{16} \mathrm{~F}_{4} \mathrm{~N}_{4}$ & $\mathrm{C}_{38} \mathrm{H}_{18} \mathrm{~F}_{2} \mathrm{~N}_{4}$ & $\mathrm{C}_{38} \mathrm{H}_{20} \mathrm{~N}_{4}$ & $\mathrm{C}_{40} \mathrm{H}_{24} \mathrm{~N}_{4}$ & $\mathrm{C}_{26} \mathrm{H}_{16}$ \\
\hline Formula weight & 604.57 & 568.58 & 532.60 & 560.66 & 328.41 \\
\hline Crystal system & Orthorhombic & Monoclinic & Monoclinic & Monoclinic & Orthorhombic \\
\hline Space group & Pbcn & $P 2_{1} / n$ & $P 2_{1} / c$ & $C 2 / c$ & $P 2{ }_{1} 2_{1} 2_{1}$ \\
\hline Crystal size, $\mathrm{mm}^{3}$ & $0.37 \times 0.32 \times 0.10$ & $0.38 \times 0.19 \times 0.11$ & $0.47 \times 0.06 \times 0.05$ & $0.49 \times 0.46 \times 0.40$ & $0.36 \times 0.24 \times 0.06$ \\
\hline$a, \AA$ & $7.1454(4)$ & $8.3747(4)$ & $8.2077(5)$ & $14.4593(7)$ & $7.3079(3)$ \\
\hline$b, \AA$ & $17.2601(9)$ & $16.7659(8)$ & $33.7880(19)$ & $11.4032(6)$ & $12.9491(6)$ \\
\hline$c, \AA$ & $22.2970(11)$ & $19.2823(9)$ & $19.3173(11)$ & $17.8273(9)$ & $17.4393(8)$ \\
\hline$\alpha, \operatorname{deg}$ & 90 & 90 & 90 & 90 & 90 \\
\hline$B$, deg & 90 & $90.488(1)$ & $92.882(1)$ & $102.652(1)$ & 90 \\
\hline$\gamma$, deg & 90 & 90 & 90 & 90 & 90 \\
\hline$V, \AA^{3}$ & $2749.9(3)$ & $2707.3(2)$ & $5350.3(5)$ & $2868.0(3)$ & $1650.3(1)$ \\
\hline$Z$ & 4 & 4 & 8 & 4 & 4 \\
\hline Temperature, $\mathrm{K}$ & 100 & 100 & 100 & 100 & 100 \\
\hline$d_{\mathrm{alc}}, \mathrm{g} \mathrm{cm}^{-3}$ & 1.460 & 1.395 & 1.322 & 1.298 & 1.322 \\
\hline$\mu(\mathrm{Mo} \mathrm{K} \alpha), \mathrm{mm}^{-1}$ & 0.106 & 0.093 & 0.079 & 0.077 & 0.075 \\
\hline Reflns used & 2811 & 5528 & 10950 & 2929 & 3371 \\
\hline Refined params & 209 & 404 & 757 & 201 & 235 \\
\hline$R_{1}(\text { for } I>2 \sigma(I))^{a}$ & 0.0321 & 0.0391 & 0.0482 & 0.0343 & 0.0294 \\
\hline$w R_{2}$ (for all data) ${ }^{b}$ & 0.0915 & 0.0991 & 0.1045 & 0.1016 & 0.0724 \\
\hline GOF on $F^{2}$ & 1.045 & 1.019 & 0.975 & 1.096 & 1.058 \\
\hline CCDC number & 1533425 & 1533426 & 1533427 & 1533428 & 1535631 \\
\hline
\end{tabular}

${ }^{a} R_{1}=\Sigma\left(\left|F_{0}\right|-\left|F_{\mathrm{c}}\right|\right) / \Sigma\left|F_{0}\right| .{ }^{b} w R_{2}=\left[\Sigma w\left(F_{0}^{2}-F_{\mathrm{c}}^{2}\right)^{2} / \Sigma w\left(F_{0}^{2}\right)^{2}\right]^{0.5}$. 
molecules (perylene, ${ }^{17}$ anthracene, ${ }^{18}$ pyrene, ${ }^{19}$ coronene, ${ }^{20,21}$ and triphenylene ${ }^{22}$ ) for their TCNQ complexes (Fig. 1b).

Infrared spectra of 1-4 are readily assigned by the superposition of those of the component molecules, which also supports the neutral ground state (Fig. S1 in ESI $\dagger$ ). For complex 1 formed with the strongest electron acceptor, $\mathrm{F}_{4} \mathrm{TCNQ}$, the $b_{1 \mathrm{u}} v_{\mathrm{C}=\mathrm{C}}$ mode $\left(1591 \mathrm{~cm}^{-1}\right)$ is shifted to a low frequency by $9 \mathrm{~cm}^{-1}$ relative to that of neutral $\mathrm{F}_{4} \mathrm{TCNQ}\left(1600 \mathrm{~cm}^{-1}\right)$. Assuming that the reduction state of $\mathrm{F}_{4} \mathrm{TCNQ}$ molecule is linearly correlated with the $v_{\mathrm{C}=\mathrm{C}}$ shift, namely $\delta=-\left(\omega_{\mathrm{obs}}\right.$ - $\left.\omega_{0}\right) /\left(\omega_{1}-\omega_{0}\right)$, where $\omega_{\mathrm{obs}}, \omega_{0}$, and $\omega_{1}$ denote the $v_{\mathrm{C}=\mathrm{C}}$ mode frequencies of a $\mathrm{CT}$ complex, neutral $\mathrm{F}_{4} \mathrm{TCNQ}$, and monoanionic $\mathrm{F}_{4} \mathrm{TCNQ}$, respectively, ${ }^{23}$ the charge of the $\mathrm{F}_{4} \mathrm{TCNQ}$ molecules in $\mathbf{1}$ was estimated to be as low as approximately -0.1 .

\section{Crystal Structure}

Crystal structures of 1-4 were determined at $100 \mathrm{~K}$ (Table 2). Despite their distinct crystal lattices, all complexes exhibit 1:1 stoichiometry, with peculiar wavy DA-type alternating $\pi$-columns. In the column, [6]helicene and TCNQ analogs are arranged in a heterochiral manner, with a $[\cdots(P)$-[6]helicene $\cdots$ TCNQ $\cdots(M)$-[6]helicene $\cdots$ TCNQ $\cdots]$ repeating unit. These complexes are racemates, and no spontaneous enantiomer resolution occurred during crystallization. Complex 3 prepared in this study exhibits a structure identical to that reported by Ermer: ${ }^{13}$ two [6] helicene and two TCNQ molecules are crystallographically independent, and the charges of the TCNQ molecules were estimated to be $-0.15(4)$ and -0.12 (3) on the basis of the intramolecular bond lengths. ${ }^{24}$

Complex 1 belongs to the orthorhombic system with space group $P b c n$, and the asymmetric unit contains a half [6]helicene and a half $\mathrm{F}_{4} \mathrm{TCNQ}$. Adjacent $(P)$-[6]helicene and $(M)$-[6]helicene within the column are related by an inversion centre, whereas $\mathrm{F}_{4} \mathrm{TCNQ}$ is located on an inversion centre. Distance between the terminal benzene centroids $(d)$ and angle between the planes of the terminal benzene rings $(\gamma)$ in [6]helicene were estimated as $4.39 \AA$ and $57.6^{\circ}$, respectively. The contraction of [6] helicene molecules in 1 relative to those in the enantiomeric crystal $\left(4.48 \AA\right.$ and $59.5^{\circ}$, respectively; Table 2$)^{25,26}$ is possibly related to Wallach's rule, ${ }^{27,28}$ which suggests denser packing in racemic crystals compared to their chiral conglomerates. From bond length analysis, ${ }^{24,29}$ the charge of $\mathrm{F}_{4} \mathrm{TCNQ}$ was estimated as $-0.05(3)$, which is in good agreement with that expected from the infrared spectrum (ca. -0.1$)$.

The complex involves wavy DA-type alternating $\pi$-columns along the $c$ axis (Fig. 2a). In the column, a terminal benzene ring of [6] helicene and a quinonoid ring of $\mathrm{F}_{4} \mathrm{TCNQ}$ exhibits a ring-overbond overlap pattern (Fig. 2b), and are arranged in a nonparallel fashion with a dihedral angle of $3.98^{\circ}$. At $z \sim 0.25$ and 0.75 , [6]helicene molecules form heterochiral sheets comprising equimolar amounts of $(P)$-[6]helicene and $(M)$-[6]helicene. Each $\mathrm{F}_{4} \mathrm{TCNQ}$ molecule is connected to two adjacent [6] helicene molecules (one $(P)$ [6] helicene and one $(M)$-[6]helicene) through $\mathrm{C}-\mathrm{H} \cdots \mathrm{F}$ hydrogen bonds $(\mathrm{H} 7 \cdots \mathrm{F} 1: 2.61 \AA, \mathrm{H} 8 \cdots \mathrm{F} 1: 2.48 \AA$ vs. sum of van der Waals radii: $2.67 \AA ; 30$ Fig. 2 c) along the molecular short axis to form an infinite array along the [lll $\left.\begin{array}{lll}4 & 1\end{array}\right]$ or $\left[\begin{array}{lll}4 & 0 & \overline{1}\end{array}\right]$ direction. In addition, each $\mathrm{F}_{4} \mathrm{TCNQ}$ molecule interacts with two adjacent [6] helicene molecules (one $(P)$-[6]helicene and one $(M)$-[6]helicene) along the molecular long axis through $\mathrm{C}-\mathrm{H} \cdots \mathrm{N}$ hydrogen bonds $(\mathrm{H} 1 \cdots \mathrm{N} 2: 2.55 \AA$ vs. sum of van der Waals radii: $2.75 \AA^{30}$ ). As shown in Fig. 2d, the hydrogen- (a)

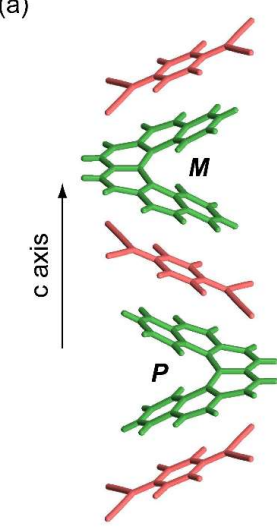

(b)

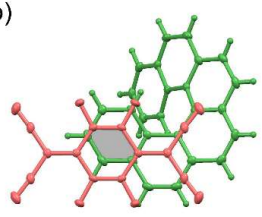

(d)

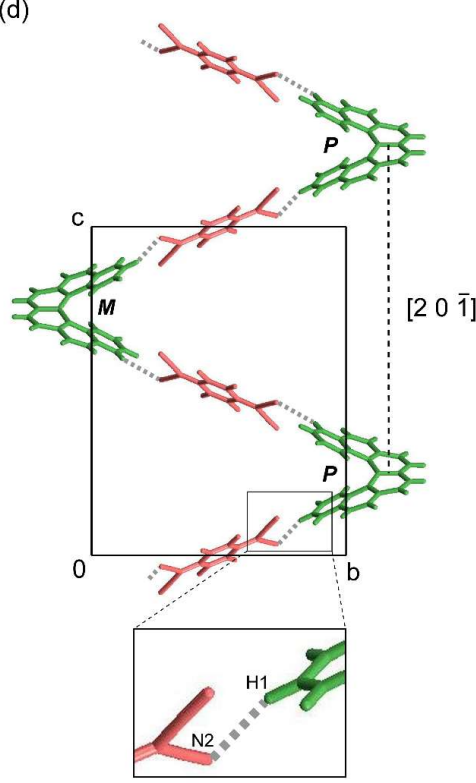

(c)

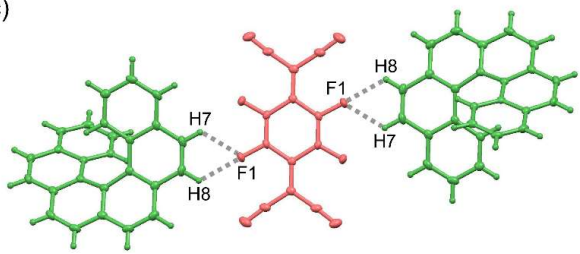

Fig. 2 (a) DA-type alternating $\pi$-column in 1 viewed along the $a$ axis, where $\boldsymbol{P}$ and $\boldsymbol{M}$ indicate $(P)$ - and $(M)$-[6] helicene molecules, respectively. (b) Overlap pattern of adjacent [6] helicene and $\mathrm{F}_{4}$ TCNQ within a column viewed perpendicular to the molecular plane of $\mathrm{F}_{4}$ TCNQ in $\mathbf{1}$. See text for the grey area. (c) Short $\mathrm{C}-\mathrm{H} \cdots \mathrm{F}$ contacts with $\mathrm{H} \cdots \mathrm{F}$ distances of less than the sum of the van der Waals radii (2.67 $\AA$; grey dotted lines) around one $\mathrm{F}_{4}$ TCNQ molecule in 1. (d) Supramolecular helix through $\mathrm{C}-\mathrm{H} \cdots \mathrm{N}$ hydrogen bonds (grey dotted lines) viewed along the $a$ axis, where a helix extending along the [ $\left[\begin{array}{lll}2 & 0 & 1\end{array}\right]$ direction is shown. [6]Helicene and $F_{4} T C N Q$ molecules are shown in green and red, respectively.

bonding interactions afford an infinite supramolecular helix with a $\left[\cdots(P)\right.$-[6]helicene $\cdots \mathrm{F}_{4} \mathrm{TCNQ} \cdots(M)$-[6] helicene $\left.\cdots \mathrm{F}_{4} \mathrm{TCNQ} \cdots\right]$ repeating unit of $26.5 \AA$ along the [2 01 l] or [ [ $\left.\begin{array}{lll}2 & 0 & 1\end{array}\right]$ direction.

Complex 2 crystallises in a monoclinic lattice with space group $P 21 / n$, and each of [6]helicene and $\mathrm{F}_{2} \mathrm{TCNQ}$ is crystallographically independent. The $d$ and $\gamma$ values of [6] helicene were estimated as 4.20 $\AA$ and $47.0^{\circ}$, respectively, and fluorine atoms in $\mathrm{F}_{2} \mathrm{TCNQ}$ were orientationally disordered over two distinct orientations, 2,5- and 3,6positions, with an occupancy ratio of 93/7.

The wavy alternating $\pi$-columns shown in Fig. 3a run along the [1 0 1] direction. Each $F_{2}$ TCNQ exhibits a ring-over-ring overlap with a second terminal benzene ring of [6] helicene with a dihedral angle of $2.32^{\circ}$ on one side, whereas it exhibits a ring-over-bond overlap on the other side (Fig. 3b). Unlike 1, [6] helicene molecules in $\mathbf{2}$ form homochiral sheets at $z \sim 0.25$ and 0.75 for $(P)$ - and $(M)$-type enantiomers, respectively, which are related by an inversion centre. Whereas fluorine atoms in $\mathrm{F}_{2} \mathrm{TCNQ}$ form no short interatomic contacts with adjacent [6] helicene molecules in contrast to $\mathbf{1}$, intermolecular $\mathrm{C}-\mathrm{H} \cdots \mathrm{N}$ hydrogen bonds $(\mathrm{H} 7 \cdots \mathrm{N} 1: 2.71 \AA$, 
(a)

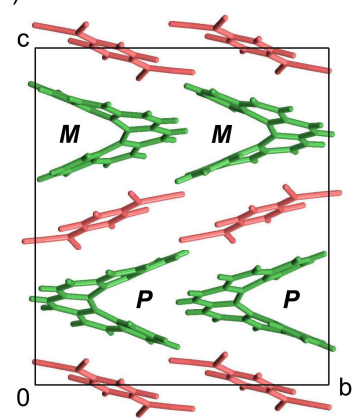

(b)

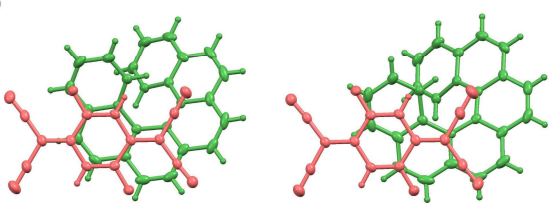

(c)

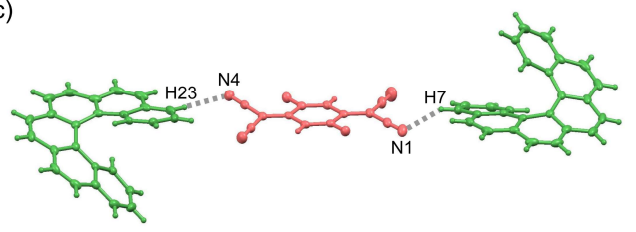

Fig. 3 (a) Crystal structure of $\mathbf{2}$ viewed along the $a$ axis, where $\boldsymbol{P}$ and $\boldsymbol{M}$ indicate $(P)$ - and $(M)$-[6] helicene molecules, respectively. (b) Overlap pattern of adjacent [6] helicene and $F_{2}$ TCNQ within a column viewed perpendicular to the molecular plane of $F_{2}$ TCNQ in 2. (c) Short $\mathrm{C}-\mathrm{H} \cdots \mathrm{N}$ contacts with $\mathrm{H} \cdots \mathrm{N}$ distances of less than the sum of the van der Waals radii ( $2.75 \AA$; grey dotted lines) around one $\mathrm{F}_{2} \mathrm{TCNQ}$ molecule in $\mathbf{2}$. Only the major orientation of the disordered $F_{2}$ TCNQ is shown, and [6] helicene and $F_{2}$ TCNQ molecules are shown in green and red, respectively.

H23 $\cdots$ N4: $2.57 \AA$; Fig. $3 c$ ) result in the formation of an infinite supramolecular helix with a $\left[\cdots(P)-[6]\right.$ helicene $\cdots \mathrm{F}_{2} \mathrm{TCNQ} \cdots(M)$ $[6]$ helicene $\left.\cdots \mathrm{F}_{2} \mathrm{TCNQ} \cdots\right]$ repeating unit of $31.5 \AA$ along the $\left[\begin{array}{lll}3 & 0 & 1\end{array}\right]$ or $\left[\begin{array}{lll}3 & 0 & 1\end{array}\right]$ direction.

Complex $\mathbf{4}$ also belongs to the monoclinic system with space group $C 2 / c$, and the asymmetric unit contains a half [6]helicene and a half $\mathrm{Me}_{2}$ TCNQ. Adjacent $(P)$-[6]helicene and $(M)$-[6]helicene within the column are related by an inversion centre and form homochiral sheets at $z \sim 0.25$ and 0.75 , respectively. Notably, the lowest $d$ and $\gamma$ values (4.12 $\AA$ and $41.8^{\circ}$, respectively) were observed.

The wavy alternating $\pi$-columns (pink area in Fig. $4 \mathrm{a}$ ) run along the $c$ axis, in which adjacent [6] helicene and $\mathrm{Me}_{2} \mathrm{TCNQ}$ molecules exhibit a ring-over-bond overlap fashion (Fig. 4b). In contrast to other complexes, a bulky methyl group in $\mathrm{Me}_{2} \mathrm{TCNQ}$, which is located on an inversion centre, approaches the adjacent helical core, affording close packing (Fig. 4c). As indicated by the blue area in Fig. 4a, this complex forms infinite supramolecular helices with a $[\cdots(P)$ [6] helicene $\cdots \mathrm{Me}_{2} \mathrm{TCNQ} \cdots(M)$-[6] helicene $\cdots \mathrm{Me}_{2} \mathrm{TCNQ} \cdots$ ] repeating unit of $17.8 \AA$ through $\mathrm{C}-\mathrm{H} \cdots \mathrm{N}$ hydrogen bonds $(\mathrm{H} 1 \cdots \mathrm{N} 1$ : $2.64 \AA$ ). Adjacent helices along the $a$ axis are connected through another $\mathrm{C}-\mathrm{H} \cdots \mathrm{N}$ hydrogen bonds $(\mathrm{H} 7 \cdots \mathrm{N} 1: 2.60 \AA)$. Considering that the helices run along the $c$ axis, each of the [6] helicene and $\mathrm{Me}_{2} \mathrm{TCNQ}$ molecules within the column comprises the same helix. (a)

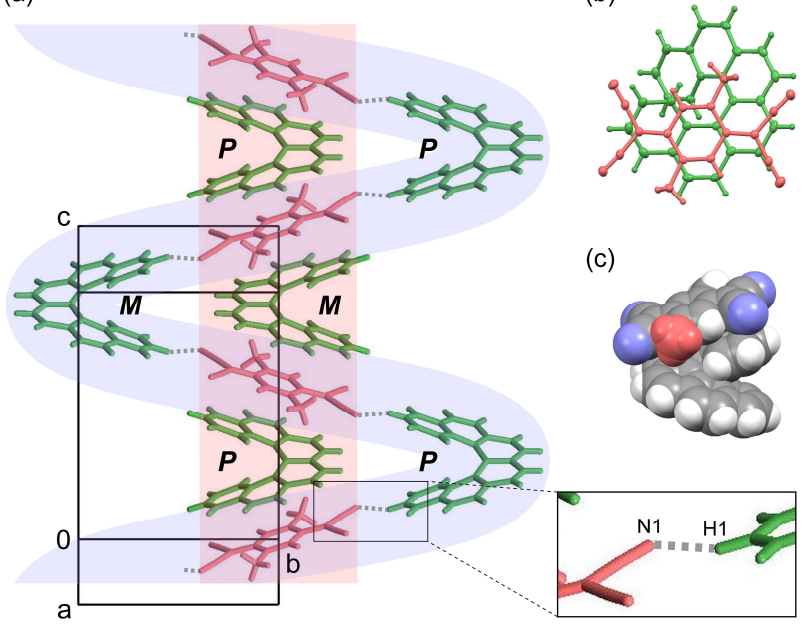

Fig. 4 (a) Crystal structure of $\mathbf{4}$, where $\boldsymbol{P}$ and $\boldsymbol{M}$ indicate $(P)$ - and (M)-[6]helicene molecules, respectively. Pink and blue areas denote the DA-type alternating $\pi$-column and supramolecular helix, respectively, both of which run along the $c$ axis. Grey dotted lines denote $\mathrm{C}-\mathrm{H} \cdots \mathrm{N}$ hydrogen bonds. (b) Overlap pattern of adjacent [6]helicene and $\mathrm{Me}_{2} \mathrm{TCNQ}$ within a column viewed perpendicular to the molecular plane of $\mathrm{Me}_{2} \mathrm{TCNQ}$ in 4. (c) Molecular packing of a pair of [6] helicene and $\mathrm{Me}_{2}$ TCNQ molecules, where a methyl group near the [6]helicene molecule is indicated in red. [6] Helicene and $\mathrm{Me}_{2} \mathrm{TCNQ}$ molecules in (a) and (b) are shown in green and red, respectively.

\section{Molecular deformation}

Thus far, the molecular geometry of [6]helicene has been modified via the coordination of a silver(I) ion $^{31}$ and the functionalization of terminal benzene rings. ${ }^{32}$ Fig. 5 shows the plots of $d$ and $\gamma$ values as a function of the $E_{1 / 2}^{1}$ (A) values of TCNQ analogs for 1-4. The [6] helicene molecules are seemingly elongated with the increase in the electron-accepting ability of TCNQ analogs; namely, intermolecular CT interactions exhibit beneficial effects on the molecular geometry of [6] helicene in the solid state. However, our density functional theory (DFT) calculations at the CAM-B3LYP$\mathrm{D} 3(\mathrm{BJ}) / \mathrm{sp}$ Aug-cc-pVDZ level of theory predicted that the cationization of $C 2$-symmetric [6]helicene marginally affects molecular geometry; the $d$ and $\gamma$ values of the [6] helicene monocation (4.21 $\AA$ and $47.2^{\circ}$, respectively) are similar to those of neutral [6]helicene ( $4.25 \AA$ and $48.5^{\circ}$, respectively)

To help understand the molecular deformation experimentally observed, we turn to the relative configuration between the benzene ring(s) of [6] helicene and a quinonoid ring of TCNQ analogs. In complex 4, a methyl group of $\mathrm{Me}_{2} \mathrm{TCNQ}$ penetrates the helical core of an adjacent [6] helicene within the column, and a hydrogen atom in the methyl group forms a $\mathrm{C}-\mathrm{H} \cdots \pi$ hydrogen bond ${ }^{33,34}$ at a distance of $2.92 \AA$ with a benzene ring of [6]helicene. Such a complementary interaction inevitably plays an important role in the close packing, possibly leading to the decreased $d$ and $\gamma$ values. On the other hand, the increased $d$ and $\gamma$ values in $\mathbf{1}$ are possibly related to the overlap ratio, which is defined as the ratio of the overlap area to the area of a quinonoid ring area viewed perpendicular to the molecular plane of TCNQ analogs (For example, a grey area shown in Fig. 2b); the 
overlap ratio in $1(54 \%)$ is apparently less than those in $2(58 \%)$ and 3 (60 and 61\%). Although the factors that determine the overlap mode in these solids remain unclear, the depressed intermolecular $\pi-\pi$ interactions between [6] helicene and F4TCNQ molecules within the column possibly result in the elongation of [6] helicene molecules in 1.

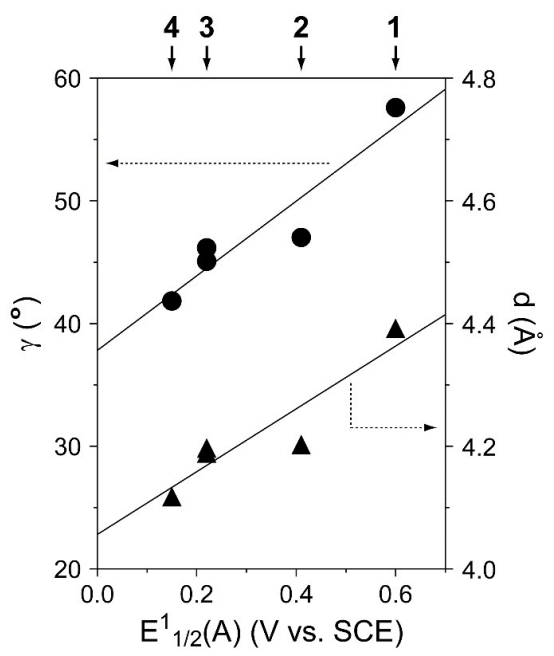

Fig. 5 Plots of distance between the terminal benzene centroids ( $d$; triangle) and angle between the planes of the terminal benzene rings $(\gamma$; circle) in a [6] helicene molecule against the first redox potential of the TCNQ analogs $\left(E_{1 / 2}^{1}(A)\right)$. Solid lines denote the least-square fits of the data.

\section{Conclusions}

The combination of racemic [6] helicene and TCNQ analogs yields racemic neutral CT complexes instead of chiral conglomerates. All complexes consist of heterochiral DA-type alternating $\pi$-columns with a wavy structure associated with the helical structure of [6]helicene. The small $\pi$ - $\pi$ overlap between the adjacent [6]helicene and $\mathrm{F}_{4} \mathrm{TCNQ}$ results in the elongation of [6]helicene, whereas the complementary interactions via $\mathrm{C}-\mathrm{H} \cdots \pi$ hydrogen bonds between [6]helicene and $\mathrm{Me}_{2} \mathrm{TCNQ}$ within the column contracts [6]helicene. Further studies will be focused on the exploration of ionic CT complexes formed with (partially) cationic [6] helicene molecules not only to construct an unprecedented higher-dimensional $\pi$-conduction network but also to elucidate the relationship between the molecular geometry of [6]helicene and electronic properties of the CT solids.

\section{Experimental section}

\section{General details}

1,1,2-Trichloroethane and chloroform were distilled from $\mathrm{CaH}_{2}$ prior to use. Racemic [6] helicene (Lach-Ner, Czech Republic) was used as received, whereas TCNQ analogs were purified by recrystallization and/or sublimation after being synthesised by our research group or purchased commercially.

\section{Syntheses of 1,2 , and 4}

Equimolar amounts of racemic [6]helicene $(0.015 \mathrm{mmol})$ and the TCNQ analog $(0.015 \mathrm{mmol})$ were ground together in an agate mortar and transferred into a borosilicate glass tube with a diameter of $12 \mathrm{~mm}$. After sealing under vacuum $\left(5 \times 10^{-5}\right.$ Torr $)$, the 13 -cm-long tube was placed in a two-zone furnace with a temperature gradient from $201{ }^{\circ} \mathrm{C}$ (sample side) to $149{ }^{\circ} \mathrm{C}$ (opposite side). After heating for 2-4 days, black thick plate crystals of $\mathbf{1}$, black hexagonal bifrustum crystals of $\mathbf{2}$, and black block crystals of $\mathbf{4}$ were grown on the opposite side in the tube. Yield: $30-50 \%$.

\section{Synthesis of 3}

Black rod crystals of $\mathbf{3}$ were obtained by the slow evaporation of a 1,1,2-trichloroethane solution $(10 \mathrm{~mL})$ containing equimolar amounts of racemic [6] helicene $(0.01 \mathrm{mmol})$ and TCNQ $(0.01 \mathrm{mmol})$. Yield: ca. $80 \%$.

\section{Spectroscopy}

UV-Vis-NIR absorption spectra were measured on $\mathrm{KBr}$ pellets $(3.8-$ $\left.42 \times 10^{3} \mathrm{~cm}^{-1}\right)$ or in a chloroform solution $\left(12-50 \times 10^{3} \mathrm{~cm}^{-1}\right)$ using a Shimadzu UV-3100 spectrophotometer. Infrared transmission spectra were measured on $\mathrm{KBr}$ pellets using a Shimadzu Prestige-21 spectrophotometer $\left(380-7800 \mathrm{~cm}^{-1}\right)$.

\section{X-Ray structural analysis}

Single-crystal X-ray diffraction experiments were performed on a CCD-type diffractometer (Bruker SMART APEX II) with graphitemonochromated Mo K $\alpha$ radiation $(\lambda=0.71073 \AA)$ at $100 \mathrm{~K}$. A single crystal was mounted on a glass capillary and cooled by a stream of cooled nitrogen gas. The crystal structures solved by a direct method using the SIR2004 program ${ }^{35}$ were refined by a full-matrix leastsquares method on $F^{2}$ using SHELXL. ${ }^{36}$ All non-hydrogen atoms were anisotropically refined. The positional parameters of the hydrogen atoms were calculated under the assumption of fixed $\mathrm{C}-\mathrm{H}$ bond lengths of 0.93 and $0.96 \AA$ with $\mathrm{sp}^{2}$ and $\mathrm{sp}^{3}$ configurations, respectively, of the parent atoms. In the refinement procedures, isotropic temperature factors with a magnitude 1.2-fold greater than that of the equivalent temperature factors of the parent atoms were applied to the hydrogen atoms.

\section{Computational details}

DFT calculations were performed using the CAM-B3LYP, ${ }^{37}$ CAMB3LYP-D3(BJ), ${ }^{37,38}$ and M11 ${ }^{39}$ functionals in combination with the spAug-cc-pVDZ basis set. ${ }^{40,41}$ Full geometry optimization, with "Opt $=$ Tight" specified, was conducted for $C_{2}$-symmetric $(P)$-[6]helicene ${ }^{0}$ as well as $C_{2}$ - and $C_{1}$-symmetric $(P)$-[6]helicene ${ }^{\bullet+}$. Note that all of the functionals found one imaginary frequency in $C_{2}$-symmetric $(P)$ [6] helicene ${ }^{\bullet+}$, indicating a transition state. The stability of the wave function was confirmed by specifying the "Stable=Opt" keyword. The "Int=SuperFineGrid" keyword was specified throughout the current DFT calculations. All computations were performed with the Gaussian 09 program package. ${ }^{42}$

\section{Acknowledgements}

This work was supported by the Japan Society for the Promotion of Science (JSPS) KAKENHI Grant Numbers JP25288041, JP26288035, JP15K17901, JP16H00964, and JP16H04139. Theoretical calculations were performed at the Research Center for Computational Science, Okazaki, Japan, and under the Collaborative 
Research Program for Young Scientists at Academic Center for Computing and Media Studies, Kyoto University.

\section{References}

1 Organic Electronics: Materials, Manufacturing, and Applications, ed. H. Klauk, Wiley-VCH, Weinheim, 2006.

2 F. S. Kim, G. Ren and S. A. Jenekhe, Chem. Mater., 2011, 23, 682-732.

3 C. Wang, H. Dong, W. Hu, Y. Liu and D. Zhu, Chem. Rev., 2012, 112, 2208-2267.

4 M. Almeida and R. T. Henriques, in Handbook of Organic Conductive Molecules and Polymers, ed. H. S. Nalwa, Wiley, New York, Vol. 1, pp.87-149, 1997.

5 D. Graf, J. S. Brooks, M. Almeida, J. C. Dias, S. Uji, T. Terashima and M. Kimata, Europhys. Lett., 2009, 85, 27009-27013.

6 G. A. Artioli and L. Malavasi, J. Mater. Chem. C, 2014, 2, $1577-1584$

7 R. Rieger and K. Müllen, J. Phys. Org. Chem., 2010, 23, 315-325.

8 Y. Segawa, H. Ito and K. Itami, Nat. Rev. Mater., 2016, 1, 15002.

9 T. Fujikawa, Y. Segawa and K. Itami, J. Am. Chem. Soc., 2016 , 138, 3587-3595.

10 Fragments of Fullerenes and Carbon Nanotubes: Designed Synthesis, Unusual Reactions, and Coordination Chemistry, ed. M. A. Petrukhina and L. T. Scott, Wiley, Hoboken, 2012.

11 Y. Shen and C.-F. Chen, Chem. Rev., 2012, 112, 1463-1535.

12 M. Gingras, G. Félix and R. Peresutti, Chem. Soc. Rev., 2013, 42, $1007-1050$

13 Y. Yoshida, K. Isomura, Y. Nakamura, H. Kishida and G. Saito, Chem. Lett., 2015, 44, 709-711.

14 O. Ermer and J. Neudörfl, Helv. Chim. Acta, 2001, 84, 1268-1313.

15 J. B. Torrance, J. E. Vazquez, J. J. Mayerle and V. Y. Lee, Phys. Rev. Lett., 1981, 46, 253-257.

16 J. W. Diesveld, J. H. Borkent and W. H. Laarhoven, Reel. Trav. Chim., 1980, 99, 391-394.

17 I. J. Tickle and C. K. Prout, J. Chem. Soc., Perkin Trans. 2, 1973, 720-723.

18 R. M. Williams and S. C. Wallwork, Acta Cryst., 1968, B24, 168174.

19 C. K. Prout, I. J. Tickle and J. D. Wright, J. Chem. Soc., Perkin Trans. 2, 1973, 528-530.

20 X. Chi, C. Besnard, V. K. Thorsmlle, V. Y. Butko, A. J. Taylor, T. Siegrist and A. P. Ramirez, Chem. Mater., 2004, 16, 57515755.

21 Y. Yoshida, Y. Shimizu, T. Yajima, G. Maruta, S. Takeda, Y. Nakano, T. Hiramatsu, H. Kageyama, H. Yamochi and G. Saito, Chem. Eur. J., 2013, 19, 12313-12324.

22 Y. Yoshida et al. unpublished data.

23 S. Horiuchi, R. Kumai, Y. Okimoto and Y. Tokura, Chem. Phys., 2006, 325, 78-91.

24 T. J. Kistenmacher, T. J. Emge, A. N. Bloch and D. O. Cowan, Acta Crystallogr. B, 1982, 38, 1193-1199.

25 B. S. Green and M. Knossow, Science, 1981, 214, 795-797.

26 S. Ramdas, J. M. Thomas, M. E. Jordan and C. J. Eckhardt, J. Phys. Chem., 1981, 85, 2421-2425.

27 O. Wallach, Justus Liebigs Ann. Chem., 1895, 286, 90-143.

28 C. P. Brock, W. B. Schweizer and J. D. Dunitz, J. Am. Chem. Soc., 1991, 113, 9811-9820.

29 B. Mahns, O. Kataeva, D. Islamov, S. Hampel, F. Steckel, C. Hess, M. Knupfer, B. Büchner, C. Himcinschi, T. Hahn, R. Renger and J. Kortus, Cryst. Growth Des., 2014, 14, 1338-1346.

30 A. Bondi, J. Phys. Chem., 1964, 68, 441-451.

31 E. Makrlík, J. J. Dytrtová, P. Vaňura, I. Císařová, J. Sýkora, V. Církva, J. Storch and M. Polášek, Struct. Chem., 2016, 27, 627635.

32 C. Wachsmann, E. Weber, M. Czugler and W. Seichter, Eur. J. Org. Chem., 2003, 2003, 2863-2876.
33 D. Braga, F. Grepioni and E. Tedesco, Organometallics, 1998, 17, 2669-2672.

34 M. Nishio, CrystEngComm, 2004, 6, 130-158.

35 M. C. Burla, R. Caliandro, M. Camalli, B. Carrozzini, G. L. Cascarano, L. De Caro, C. Giacovazzo, G. Polidori and R. Spagna, J. Appl. Crystallogr., 2005, 38, 381-388.

36 G. M. Sheldrick, SHELXL-2013, University of Göttingen, Germany, 2013.

37 T. Yanai, D. Tew and N. Handy, Chem. Phys. Lett., 2004, 393, $51-57$.

38 S. Grimme, S. Ehrlich and L. Goerigk, J. Comp. Chem., 2011, 32, $1456-1465$.

39 R. Peverati and D. G. Truhlar, J. Phys. Chem. Lett., 2011, 2, 28102817.

40 T. H. Dunning, Jr., J. Chem. Phys., 1989, 90, 1007-1023.

41 R. A. Kendall, T. H. Dunning, Jr. and R. J. Harrison, J. Chem. Phys., 1992, 96, 6796-6806.

42 M. J. Frisch, G. W. Trucks, H. B. Schlegel, G. E. Scuseria, M. A. Robb, J. R. Cheeseman, G. Scalmani, V. Barone, B. Mennucci, G. A. Petersson, H. Nakatsuji, M. Caricato, X. Li, H. P. Hratchian, A. F. Izmaylov, J. Bloino, G. Zheng, J. L. Sonnenberg, M. Hada, M. Ehara, K. Toyota, R. Fukuda, J. Hasegawa, M. Ishida, T. Nakajima, Y. Honda, O. Kitao, H. Nakai, T. Vreven, J. A. Montgomery Jr, J. E. Peralta, F. Ogliaro, M. Bearpark, J. J. Heyd, E. Brothers, K. N. Kudin, V. N. Staroverov, R. Kobayashi, J. Normand, K. Raghavachari, A. Rendell, J. C. Burant, S. S. Iyengar, J. Tomasi, M. Cossi, N. Rega, J. M. Millam, M. Klene, J. E. Knox, J. B. Cross, V. Bakken, C. Adamo, J. Jaramillo, R. Gomperts, R. E. Stratmann, O. Yazyev, A. J. Austin, R. Cammi, C. Pomelli, J. W. Ochterski, R. L. Martin, K. Morokuma, V. G. Zakrzewski, G. A. Voth, P. Salvador, J. J. Dannenberg, S. Dapprich, A. D. Daniels, Ö. Farkas, J. B. Foresman, J. V. Ortiz, J. Cioslowski and D. J. Fox, Gaussian 09 (Revision E.01), Gaussian Inc., Wallingford CT, 2013. 